\title{
The Story of the End of the World
}

\author{
by Michael Shea
}

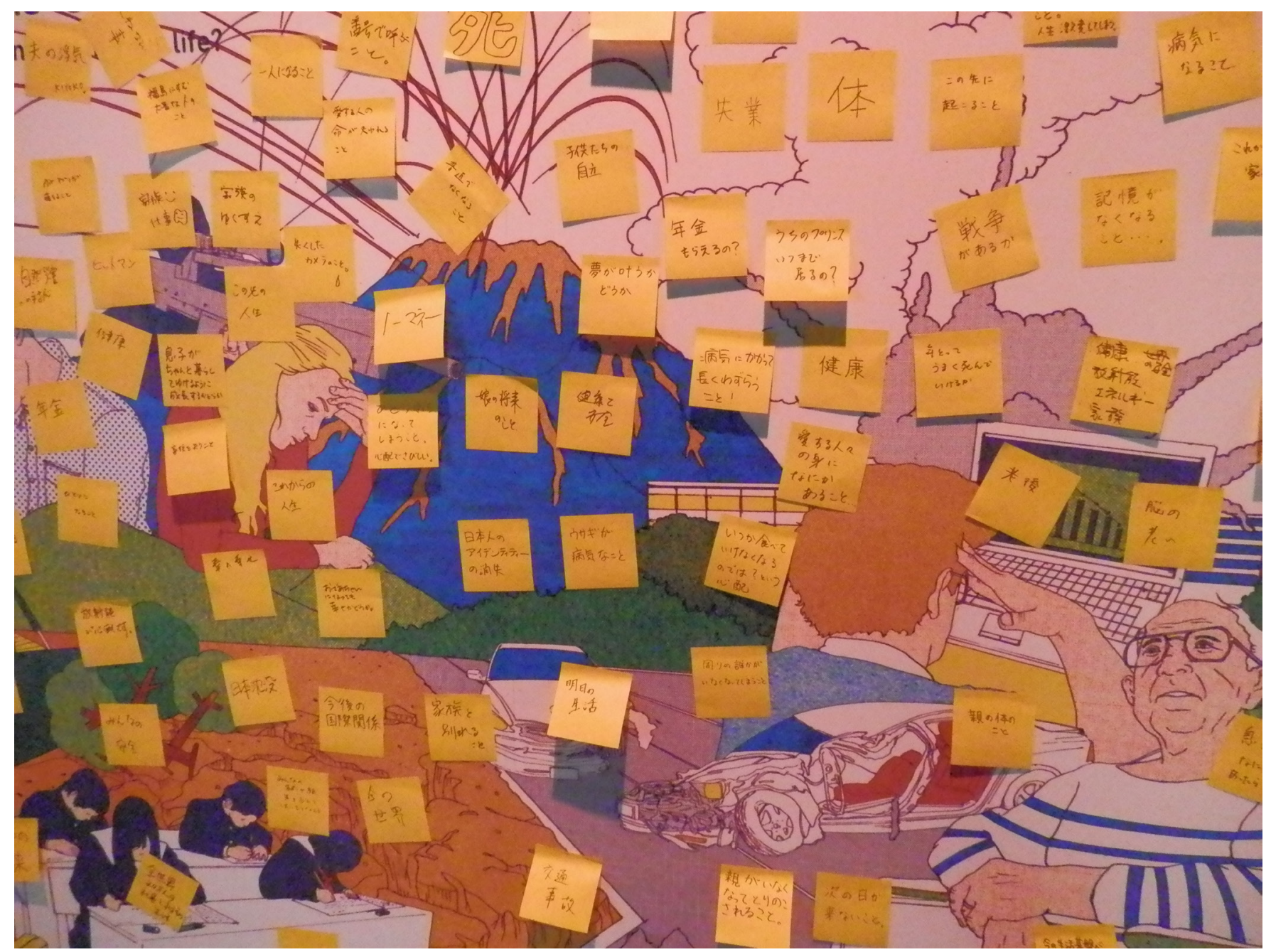

Post-it notes in response to questions from the exhibition The Story of the End of the World 


\title{
The Story of the End of the World:An Alternative Approach to the Future at the Japanese Museum of Science and Innovation
}

\author{
Michael Shea
}

The following discussion is a critical assessment of the "Story of the End of the World: 73 Questions We Must Answer", an exhibition at the Miraikan. The Tohoku earthquake and tsunami on the II th of March 201 I created the necessity for collective catharsis regarding various threats posed by the future. I will argue that the outcome of the exhibition differs significantly in content, presentation and meaning to the more conventional visions of the future presented elsewhere in the museum.

The Japanese National Museum of Emerging Science and Innovation in Tokyo, known as Miraikan, where I conducted my doctoral research, was designed to be a forum for visitors; a place where people come to engage with the future in science and technologies. The future is embedded into the design and location of the museum in several ways. The museum, which opened in July $200 \mathrm{I}$ in Odaiba, is located on an artificial island connected to the mainland accessed by an electronic railway. Miraikan's building looms around empty space allocated for future construction, semi-completed office buildings, hotels and shopping malls. The museum is vast in scale, with glass walls, a slanted ceiling and a globe representing the earth. The greeters located by the entrance, wear grey Star Trek inspired jumpsuits. By the time a visitor sets foot in the museum the future is already established as the mis-en-scene.

On the $\mathrm{II}^{\text {th }}$ of March $20 \mathrm{II}$, an earthquake shook Miraikan to its foundations. Large sections of the interior ceiling in its six-story atrium collapsed. Fortunately no one was hurt. The museum was closed for three months. A small display by the main staircase is explaining how the plasterboard ceiling has been replaced by glass fiber textile, a more durable but lightweight substance, causing less damage if it were to collapse again. There is a permanent exhibit that graphically illustrates thousands of small earthquakes occurring on a map of Japan in real time. This shows the country to be gently vibrating constantly. The volunteers sometimes use the Tohoku earthquake as a point of comparison when explaining how small and relatively insignificant these more frequent seismic events are. The GEO-cosmos, a giant LCD globe that displays various kinds of geographic information, demonstrates the impact of the Tohoku earthquake on a global scale.

As an institution that facilitates public engagement with science and nature, Miraikan was compelled to respond to the events of March 20I I. During the museum's closure, the staff had been inundated with questions regarding the future threat of earthquakes and the chances of survival in the event of another large-scale disaster. The most direct reply to the catastrophe is currently found in The Story of the End of the World (seikai no owari no monogatari) temporary exhibition. which took place between March and June 20I2. The exhibition opened on the $10^{\text {th }}$ of March, the day before the first anniversary of the earthquake.

I will argue that this exhibition can be seen as a means of achieving catharsis in regards to the Tohoku earthquake and as a potential for similar such disasters in the future. Its tone and content are significantly different to the visions of the future presented elsewhere in the museum that broadly can be characterized as techno-utopian. The following discussion will consider the exhibition content along with visitor's responses in order to argue that it constitutes an alternative approach to the future. In the interest of clarity, when the term "catharsis" is used in this discussion, it is not intended in the Freudian sense, by which repressed thoughts that are confronted but rather in a philosophical sense, as a process of intellectual cleansing through the recognition of negative emotions. In these terms the exhibition is cathartic in that it encourages the visitors to confront, articulate, and come to terms with their feelings towards death.

\section{An Alternative Approach to the Future}

The purpose of The Story of the End of the World exhibition is described on its accompanying webpage as follows:

Everything comes to an end. Human lives, nature, civilization - even our universe. In spite of this, our busy schedules have left us little room for contemplation of "endings." The disastrous Great East Japan 
Earthquake of March II, 20I I, reminded us that even the most peaceful, tranquil days may be brought to an end.We have come to the realization that our lives, bolstered by science and technology, are frail and fleeting. In light of the immediacy of such "endings," let us consider what we should cherish in our lives and how we should live side-by-side with science and technology ... Out of this knowledge of "endings," we will find the hope to keep on living (Exhibition Outline, Miraikan 20I2).

The exhibition is essentially an exploration of mortality through various existential questions: "What do you want to do during the time you have left?" "What are there [sic] in systems of life that continue forever?" etc. These questions are posted throughout the exhibition space designed to be interactive as there are pens, paper and magnets scattered everywhere for visitors to use. Some of the questions are accompanied by a notice board where visitors are encouraged to post their responses. Others respond through surveys, answering questions such as "Which is a bigger fear for you; acts by humans or acts by nature?" Underneath this question are two columns and by placing a yellow magnet under one of the columns, visitors' can register their vote (acts by humans was seen as more frightening with a ratio of 3:I). These interactive systems for measuring visitors' opinions are partly inspired by similar chalkboard-based forums posted throughout the permanent exhibitions. The walls of hand-written post-it notes create a powerful visual representation of visitors' attitudes and opinions.

The subject of the exhibition was inspired by the disaster and its impact on the museum and its staff. As a result, there is a subtle humour in which several of the staff approached the threat of earthquakes. In one volunteer meeting, the speaker joked that in the event of an earthquake, you should either help others or take off your staff jacket so that you couldn't be identified as you ran away. In my time at the museum, I only met one staff member who was openly upset about the threat of earthquakes. This individual was visibly frightened the morning after another small earthquake and told me that she "can't handle them". To promote the exhibition some of the Science Communicators, and educators at the museum, wear cone shaped hats, each displaying one of the $\mathbf{7 3}$ questions displayed at the core of the exhibition. This is a strange choice as the questions are often serious, morbid or open-ended provoking a bemused reaction from many visitors. The appearance of a friendly stranger wearing a dunce hat that says, "What do you fear the most?" or some other profound personal question is a little unnerving to say the least.

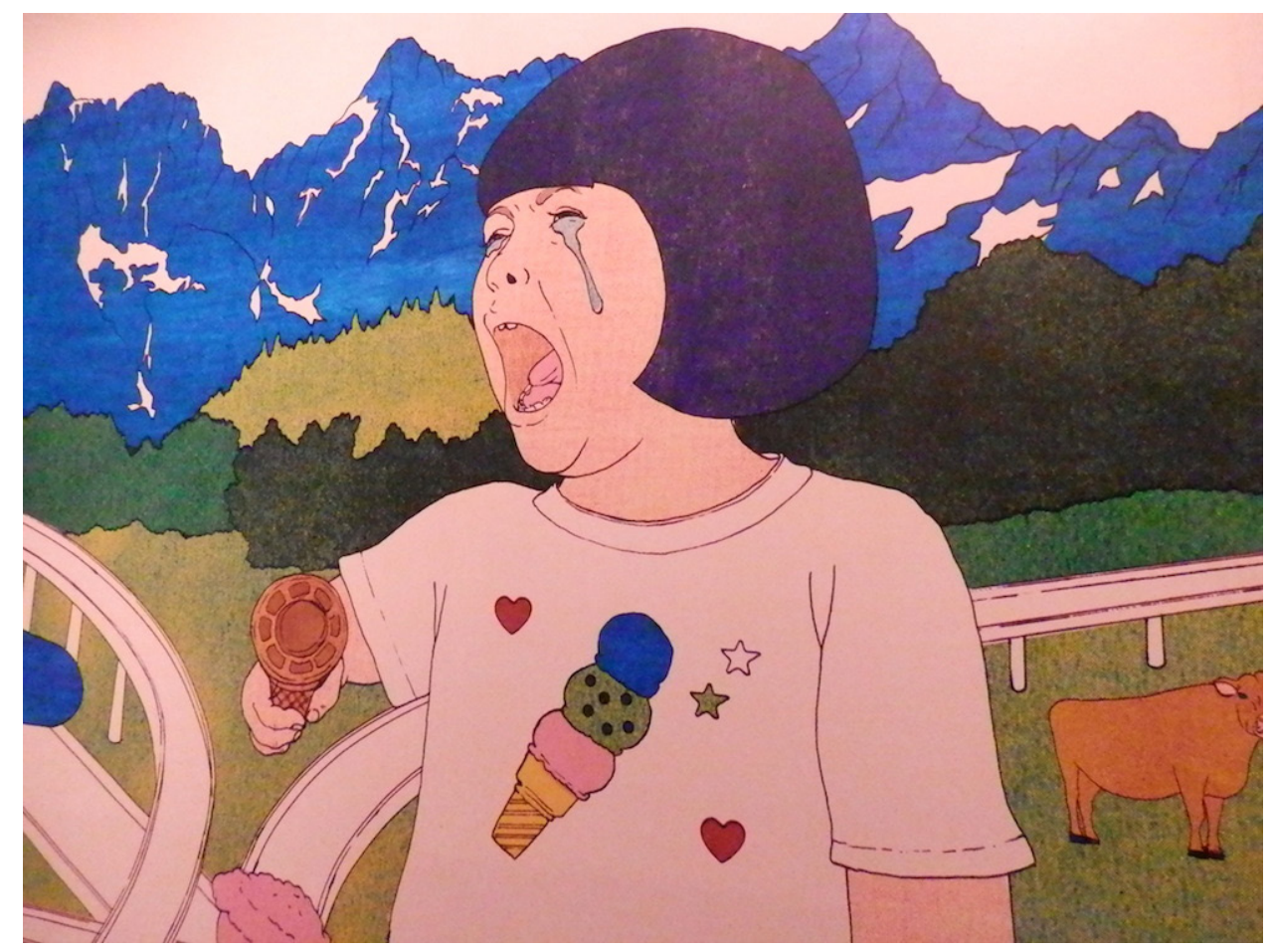

Fig. I A Story of the End of the World wall poster by Takashi Taima 
In terms of content, the exhibition is unusual as the 73 questions themselves are the main substance of the visitors' experience. As a science and technology museum, most exhibits at Miraikan involve an interactive component (touch screens, joy sticks, switches, buttons etc). Yet, in The Story of the End of the World most of the content is textual. The visitors make their way through a maze of existential questions. The questions are written on pyramid shaped boards that are lit with spotlights, the exhibition space is low lit and unadorned giving an overall impression similar to a contemporary art gallery. It seems to have been intentionally created as a contemplative space. This similarity is also highlighted by the exhibition's accompanying artwork, designed by Takashi Taima, a well-known illustrator, who created his own vision of the end of the world through a series of vibrant and nostalgic images that covered the walls at the entrance to the exhibition space.

Post-it notes for writing responses to the exhibition questions constituted an interactive component, albeit a decidedly low-tech one. Although, the exhibition did include an accompanying website where visitors could post their responses and a computer terminal, which cheerfully offered to calculate the visitors' remaining lifespan. One of the first questions that a visitor would encounter is "What is the thing you are most worried about in life?" with space below for visitors to post their replies. It provoked an impressive variety of responses. I noticed that someone had written "rice" (gohan); someone had drawn the character for "death" (shi) while another incorporated a picture of a dead body. Some other memorable responses included:"being alone," "tomorrow's life," "if the next day doesn't come," "being separated from family," "getting sick," "if my rabbit gets sick," "hitmen," "no money." With an array of material displayed including educational content, the majority of which is in the form of statistics; a model graphically represents the likely causes of death in each region of the world, as well as a display in which countries in the world are ranked by their suicide rate. Contrary to popular belief, Japan is not the country with the world's highest suicide rate, although it is ranked second in Asia behind South Korea.

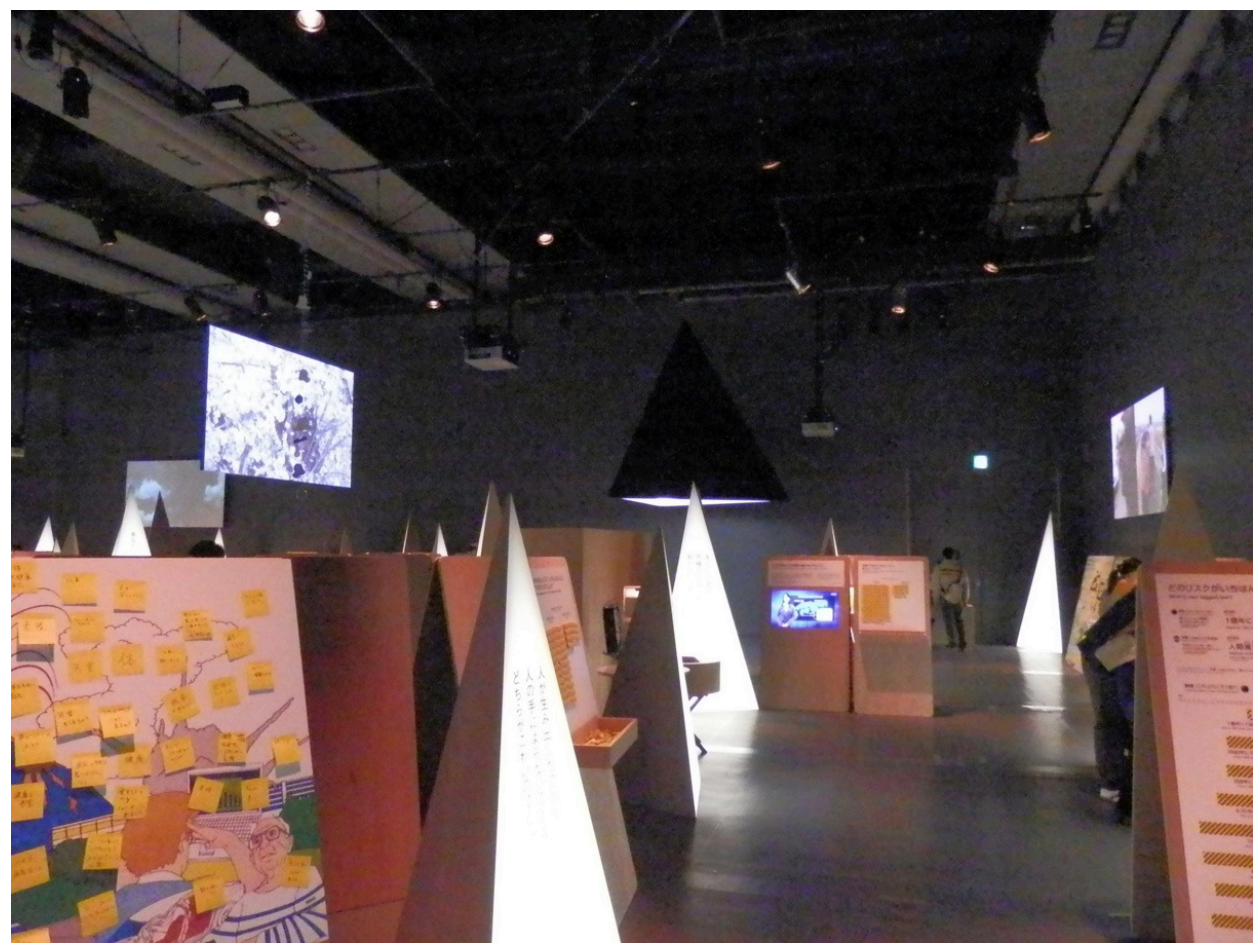

Fig.2 Inside The Story of the End of the World exhibition space

There is a similar set of rankings by country for life expectancy, displayed in the form of a line graph. Here, Japan is at the top, "Japan is amazing..." (nihon wa sugoi...) a visitor to the left of me once remarked, before asking why it was that life expectancy for women was uniformly higher than men, as we examined the graph together. This comparative approach is interesting as one way of interpreting various kinds of threats is to rationalize the relative likelihood of their occurrence. A Japanese person might be relieved to see that life 
expectancy is so high, but alarmed that the threat of natural disasters is much worse than in most other countries. It goes without saying that mortality is a consistent theme among most of the data and questions that form the exhibition.

Mortality poses a particular problem for a museum that promotes emerging science and technology, as it is not a disease to be cured or a social problem to be resolved. It cannot be fixed, which makes it somewhat of an anomaly in a science museum that elsewhere seeks to set out how technology has and will continue to find all manner of solutions. While beliefs over the potential of spiritual immortality differ from person to be person, in Western scientific thought, the dominant belief system at Miraikan, mortality cannot be defeated, only confronted and accepted. The March $\mathrm{II}^{\text {th }}$ earthquake forced the museum to respond to the inevitability of death in the only way that it could, through rationalization.

The responses to the questions are at times both humorous and moving. One sign asks: "Are there things that have disappeared due to technological advancement?" The responses include:"waste," "guts," "wasting time," "considerateness," "the self." Another asks "What do you want to do during the time left to you?" "sleep," "smile," "laugh," "it depends how long is left," "spend time with the people that are important," "to live normally." Compared to the utopian visions prevalent in many representations of the future elsewhere in the museum, which presents visions that seek to pose solutions to current problems, this exhibition is quite unusual in that it offers no solutions. To say that the exhibition is influenced by the social climate in Japan would be an understatement. It is a direct response to the devastating events in the recent past, both retrospectively orientated and presenting a somewhat fatalist vision of the future.

The exhibition is divided into four main sections: Unanticipated Endings, My End, the End of Culture and the End of the Story. In the End of Culture section there is a timeline that charts the rise and fall of various extinct civilizations. The exhibition considers the impending threat of climate change along with its potential for society. One question, which relates earlier, is: "Can we return to the lifestyle of fifty years ago?" This question is an inversion of a question found throughout the rest of the museum: "What will society look like fifty years in the future?"

On the third floor of Miraikan, there is an exhibit titled Lifestyle 2050 that consists of a scale model of an imaginary future city, Itookashi. Interactive terminals present heavily stylized cartoonish depictions of the year 2050 that resemble The Jetsons. For instance, the residents of this future city travel around in multi-colored egg shaped helicopters that suspiciously resemble the iconic 1960s chairs of the same shape. The Lifestyle 2050 exhibit is not a serious attempt at sooth-saying but rather a playful representation of a utopia based on current social concerns; an example of "future nostalgia" (Rosenberg and Harding 2005). Energy, transport, and climate needs, are fulfilled in this city, the residents are happy flying around in their eggs, the model incorporates lots of green and open space and looks significantly less crowded and congested than Tokyo, as you would expect in an ideal city. The choice of 2050 as the determinate year is interesting. The arbitrary significance of this year is that it is roughly half a century away. It is a year that a Japanese person under 40 should expect to live to see. Japan, which has long been celebrated for the longevity of its population, continues to rank first in the world with a current life expectancy of 84 (World Health Organization 20I4).

The year 2050 recurs many times throughout the museum in order to represent a notion of the future. A chalkboard invites visitors to suggest what they think Christmas gifts will be in the year 2050. The question on the board changes daily but often includes this year. Before starting in the field, I was already familiar with this year, by which the proportion of the population over 65 is expected to reach $40 \%$. This impressive life expectancy, coupled with a low birth rate, has led to an aging population, an increase cause for concern. Fears that the working population might soon no longer have the resources to support the elderly both financially or simply in support labor, can be seen in new government policies, such as the Long Term Nursing Insurance that asks individuals to contribute towards the care assistance they may depend on in later life. These fears are put into context when you consider that the population of Japan is expected to shrink by 40 million in the year 2055 , from 127.8 million to 89.93 million. With such evidence, there is an increasing sense of urgency concerning how to deal with the aging population. 


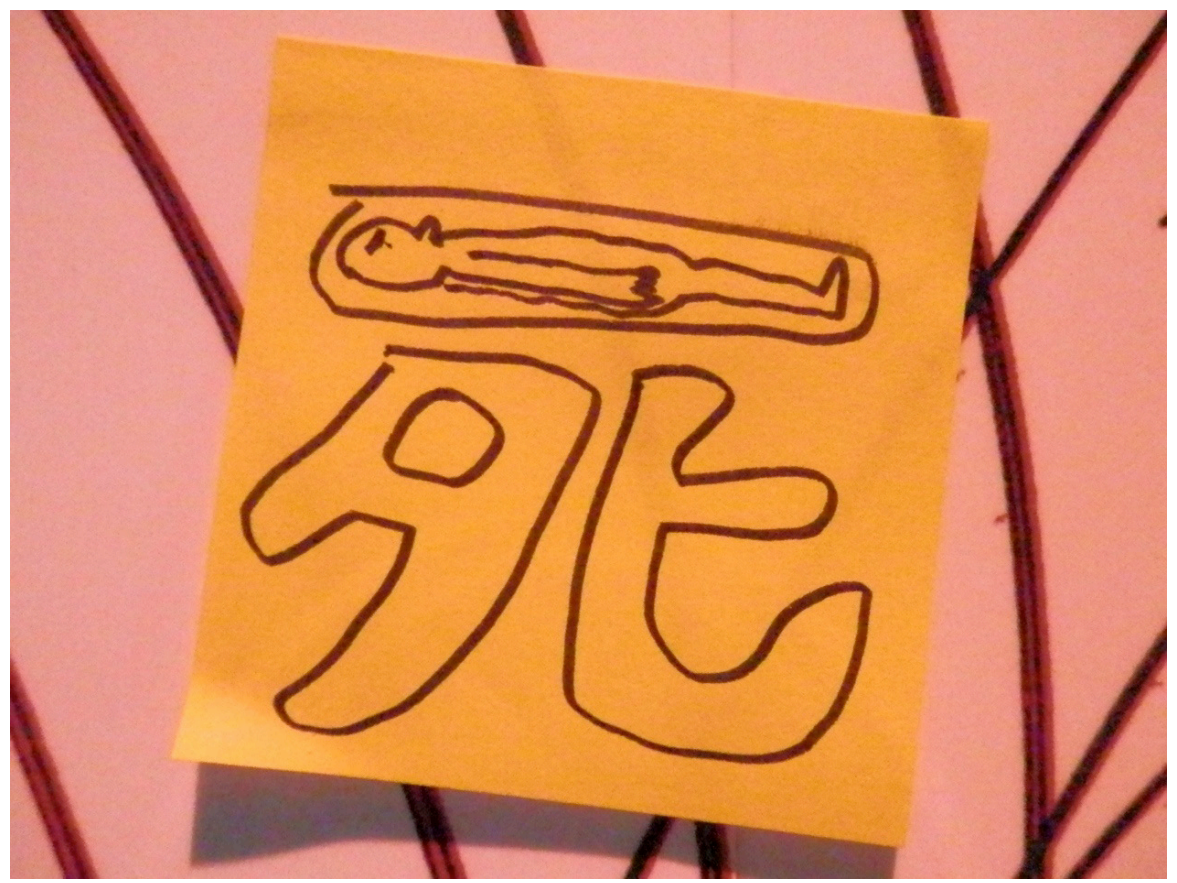

Fig.3 The character for death on a post-it note in response to the question "What is the thing you are most worried about in life?"

Advances in technology are looked to as a potential social savior for the country. For example, the government led initiative Innovation 25, announced in 2007, that Prime Minister Abe's plan for remaking Japanese society by the year 2025 was based around the increasing integration of domestic androids. Writing on this proposal, Robertson describes how Innovation 25 "emphasizes the central role that household robots will play in stabilizing core institutions, like the family" (Robertson 2007: 370). In my own research into demonstrations of the Honda ASIMO robot, the actions the android would frequently perform - carrying a tray of drinks up and down the stairs, and responding to voice commands - takes on a much greater significance once you consider that its designers explicitly refer to home care as one of it's most likely future uses.

In Boia's Forever Young:A Cultural History of Aging it is argued that since the advent of modernity, a kind of "secularized religion", has emerged whereby mortality is transcended not through religious salvation, but through notions of collective progress and nationhood. The logic underlying this shift is characterized as follows: "Each of us is mortal, but humanity will live on. The only way to give meaning to our lives is to devote ourselves to some grand collective project" (Boia 2004: 7). In science museums, this ethos can be seen in the potential for a better standard of living in the future provided by technology. The story of the end of the world exhibition becomes significant because it goes against this dominant trope that instead question the uncertainty of life in the future, natural disasters and the limit of humans potential to solve questions of life and death. The overwhelmingly optimistic ethos of technology as savior which is exemplified in Lifestyle 2050 is a world away from The Story of the End of the World, which approaches the same impeding challenges with a sober questioning of whether even the lifestyle of the immediate past, can endure. This huge shift of emphasis in the approach to the future reflects the sense of uncertainty following the $\mathrm{II}^{\text {th }}$ of March $20 \mathrm{II}$ earthquake and leads visitors to ask, if we might return to the lifestyle of fifty years ago, or at least sustain current living standards, rather than, to be invited to ponder what a marvelous place the future might be.

\section{The Uncertainty of the Future: Concluding Reflections}

Predictions about the future will always to some extent "re-inscribe the present into the future" (Collins 2008: 89). Although differing in their approaches, both the optimistic vision of the future set out in Lifestyle 2050 and The Story of the End of the World, with its existentialist musings, similarly depends on projecting present social anxieties into the future. While elsewhere in the museum the challenges of an aging population are presented in terms of the emancipatory potential of assistive technologies, the root concern with the challenge of longevity 
is the same. The Tohoku earthquake has arguably altered attitudes towards these perceived future challenges from an emphasis on improving quality of life to one of survival. The question of earthquakes might have been approached as how we might one day be able to accurately predict them. This exhibition reframes the problem by questioning: what are the odds of dying in an earthquake? Can earthquakes ever be predicted? It is about achieving catharsis by confronting uncertain and uncontrollable things.

All of the exhibits at Miraikan are maintained by the combined efforts of professional staff and volunteers. Volunteers at the museum tend to be people that have retired. It is interesting, then, to consider that many of the themes being played out in the exhibition are also directly relevant to the volunteers, mortality, future uncertainty, changes in society due to technology and their potential benefits/losses. It is of course ironic that retired volunteers, who offer up their labour for free, largely maintain an exhibit inspired by the impending crisis of supporting an aging population.

A member of staff once lost patience with me for accidentally referring to the exhibition as "the end of life" rather than "the end of the world". However, this slip of the tongue led me to consider the fact that my mistaken title would have been quite appropriate. In the exhibition, there are few, if any, scenarios presented where our planet and all life on Earth would be destroyed. Instead we are met with statistics on national suicide rates and the life span of various mammals, along with different forums for visitors to express their individual anxieties about their own future and mortality. It is really an exhibition about coming to terms with death and loss rather than an attempt to imagine the destruction of Earth. Although, nuclear war and environmental issues, that could potentially cause an apocalypse, can be seen among visitors' concerns. Questions such as "What would you like to do with the rest of your life?" are intended to encourage visitors to ponder their own life rather than to present them with a vision of a future apocalypse.

What singles out The Story of the End of the World among Miraikan exhibits, that present visions of the future in which missing workers and kin are replaced through technological innovation, is that here the loss is never compensated. This loss is felt even in the structure of the exhibition space itself; there is no central object of curiosity or technological sophistication to gaze at or interact with. One could argue that absence is at the very heart of this exhibit. In the wake of the Tohoku earthquake and tsunami there is no room for unbridled optimism. Instead, the museum presents an uncertain vision of the immediate future in which lost social relations are not easily replaced. In this exhibition, the only thing that can fill the void is the visitors and their thoughts. Rather than being faced with a technological utopia or even the destruction of Earth, the visitors are confronted with the uncertainty of the future and the inevitability of their own mortality.

\section{References}

Boia, L. (2004) Forever Young:A Cultural History of Aging. London: Reaktion.

Collins, S. G. (2008) All Tomorrow's Cultures:Anthropological Engagements with the Future. New York: Berghahn.

Miraikan Online. (20I2) 'The Story of the End of the World: Exhibition Outline'. Available at http://www.miraikan.jp/sekainoowari/outline/ [Accessed 29 September 20I4].

Robertson, J. (2007) 'Robo Sapiens Japanicus: Humanoid Robots and the Posthuman Family' Critical Asian Studies. 39 (3) 369-398.

Rosenberg, D., Harding, S. (2005) Histories of the Future. Durham: Duke University Press.

World Health Organization. (2014) Life Expectancy at Birth: Both Sexes, World Health Statistics. Available at http://gamapserver.who.int/gho/interactive_charts/mbd/life_expectancy/atlas.html [Accessed 26 August 2014]. 